\title{
Ferulic acid production by metabolically engineered Escherichia coli
}

\author{
Huajun Lv ${ }^{1 \dagger}$, Ying Zhang ${ }^{1,2+}$, Jie Shao ${ }^{1,2}$, Haili Liu' ${ }^{1}$ and Yong Wang ${ }^{1 *}$ (B)
}

\begin{abstract}
Ferulic acid ( $p$-hydroxy-3-methoxycinnamic acid, FA) is a natural active substance present in plant cell walls, with antioxidant, anticancer, antithrombotic and other properties; it is widely used in medicine, food, and cosmetics. Production of FA by eco-friendly bioprocess is of great potential. In this study, FA was biosynthesized by metabolically engineered Escherichia coli. As the first step, the genes tal (encoding tyrosine ammonia-lyase, RsTAL) from Rhodobacter sphaeroides, sam5 (encoding p-coumarate 3-hydroxylase, SeSAM5) from Saccharothrix espanaensis and comt (encoding Caffeic acid O-methytransferase, TaCM) from Triticum aestivum were cloned in an operon on the pET plasmid backbone, E. coli strain containing this construction was proved to produce FA from L-tyrosine successfully, and confirmed the function of TaCM as caffeic acid O-methytransferase. Fermentation result revealed JM109(DE3) as a more suitable host cell for FA production than BL21(DE3). After that the genes expression strength of FA pathway were optimized by tuning of promoter strength (T7 promoter or T5 promoter) and copy number (pBR322 or p15A), and the combination p15a-T5 works best. To further improve FA production, E. coli native pntAB, encoding pyridine nucleotide transhydrogenase, was selected from five NADPH regeneration genes to supplement redox cofactor NADPH for converting $p$-coumaric acid into caffeic acid in FA biosynthesis process. Sequentially, to further convert caffeic acid into FA, a non-native methionine kinase (MetK from Streptomyces spectabilis) was also overexpressed. Based on the flask fermentation data which show that the engineered $E$. coli strain produced $212 \mathrm{mg} / \mathrm{L}$ of FA with $11.8 \mathrm{mg} / \mathrm{L}$ caffeic acid residue, it could be concluded that it is the highest yield of FA achieved by E. coli K-12 strains reported to the best of our knowledge.
\end{abstract}

Keywords: Ferulic acid, E. coli, Biosynthesis, NADPH, SAM

\section{Introduction}

FA is a ubiquitous phenolic acid naturally presents in plant cell walls, mainly cross linked with polysaccharides and lignin (Harris and Trethewey 2009). It is the main effective component of Angelica sinensis, Ligusticum chuanxiong Hort., Ferula foetida and other traditional Chinese medicinal herbs (Liang et al. 2018). FA is an important plant antioxidant that acts as a free radical scavenger. Additionally, its antimicrobial,

\footnotetext{
*Correspondence: yongwang@cemps.ac.cn

${ }^{\dagger}$ Huajun Lv and Ying Zhang contributed equally to this work

${ }^{1}$ CAS Key Laboratory of Synthetic Biology, CAS Center for Excellence in Molecular Plant Sciences, Shanghai Institute of Plant Physiology and Ecology, Chinese Academy of Sciences, Shanghai 200032, China Full list of author information is available at the end of the article
}

anti-inflammatory, anticancer, antithrombotic and other pharmaceutical activities have led to the widespread use of FA in food, cosmetics and medicine (Sgarbossa et al. 2015; Wen and Ushio 2017). Extracting FA through alkaline hydrolysis or esterase treatment of plant materials rich in FA, such as Angelica sinensis, Ferula, Ligusticum chuanxiong Hort., wheat and rice bran, are the main sources of commercial FA. However, these processes require costly and challenging membrane separation during downstream processing.

Plant tissue culture can provide higher yields than extraction from plant material, but this process generates pollution and there is still no industrial scale-up. Chemical synthesis of FA from vanillin via the Wittig-Horner reaction or Knoevenagel reaction offer a short producing 
cycle, low cost, and large output, but these approaches produce a mixture of trans- and cis-ferulic acids, which are challenging to separate.

Microbial approaches provide a promising alternative to chemical synthesis and extraction from plant sources. Products obtained through biotechnological processes from natural substrates are considered as natural for the purposes of product labeling (Serra et al. 2005). With the approval of such products by the FDA and European legislation, many studies are focused on approaches based on biotechnological methods for the production of flavors, fragrances, and pharmaceutical products (Fowler and Koffas 2009; Goris et al. 2020; Luziatelli et al. 2019). There are many reports on the heterologous biosynthesis of FA-related natural products such as $p$-coumaric acid, caffeic acid, vanillin, and curcuminoids (Braga and Faria 2020; Jendresen et al. 2015; Rodrigues et al. 2015b), but there are few papers and patents on the biosynthesis of FA (Additional file 1: Table S1). Choi et al. obtained an FA titer of $7.1 \pm 1.3 \mathrm{mg} / \mathrm{L}$ via the expression of tyrosine ammonia-lyase and 4-coumarate 3-hydroxylase from $S$. espanaensis, together with $O$-methyltransferase from Arabidopsis thaliana. Tyrosine ammonia-lyase catalyzes the non-oxidative deamination of the primary amino acid tyrosine into $p$-coumaric acid, which is converted into caffeic acid by $p$-coumarate 3 -hydroxylase. Finally, FA is biosynthesized from caffeic acid by the enzyme caffeine $O$-methyltransferase. Consequently, tyrosine is transformed into ferulic acid (Choi et al. 2011). Kang et al. expanded on this work by constructing a chromosomally engineered tyrosine over-producing strain based on $E$. coli $\mathrm{C} 41$ (DE3) to explore the potential of FA production from simple carbon sources. After additional codon optimization of the tal gene, the titer of FA was increased to $196 \mathrm{mg} / \mathrm{L}$ (Kang et al. 2012).

Heterologous biosynthesis of FA in E. coli relies on multiple aspects, including the tuning of the expression levels of pathway enzymes, optimizing the supply of redox cofactors and precursors, and also the external addition of tyrosine.

A high gene copy number is generally desired for maximal expression, but the resulting metabolic burden can reduce the overall productivity in certain metabolic engineering applications. Similarly, choosing suitable promoter strength is also an effective way to obtain high yields. The combination of promoters and gene copynumbers to modulate the expression levels of up and downstream pathways resulted in a remarkable 15,000fold increase of the taxadiene titer in E. coli (Ajikumar et al. 2010; Jones et al. 2000; Ni et al. 2015). It is convenient to tune the expression levels of pathway genes using different combinations of plasmid replicon and promoter strengths.
In the FA biosynthesis pathway, the p-coumarate 3-hydroxylase, SAM5, converts $p$-coumaric acid into caffeic acid and consumes NADPH (Nicotinamide adenine dinucleotide phosphate) at the same time. Accordingly, numerous studies have shown that increasing the NADPH regeneration rate could increase both the pathway productivity and product yield. Based on a rationally engineered heterologous Entner-Doudoroff pathway that could increase the NADPH regeneration rate in $E$. coli MG1655, the product titer of the carotenoid biosynthesis pathway was increased by $97 \%$ (Martinez et al. 2008; Ng et al. 2015). The pentose phosphate pathway (PPP), the tricarboxylic acid (TCA) cycle and the transhydrogenase system constitute the main sources of NADPH (Sauer et al. 2004). The NADPH/NADP ${ }^{+}$ratio in E. coli can be enhanced to varying degrees by overexpressing several $\mathrm{NADPH}$ regeneration enzymes. NADPH is required for the synthesis of the key fucosyllactose precursorguanosine $5^{\prime}$-diphosphate (GDP)- $L$-fucose. Intracellular redox regeneration pathways were engineered to further enhance the production of 2- and 3-fucosyllactose (Huang et al. 2017), and these gene engineering approaches may also be effective for the heterologous biosynthesis of FA.

Additionally, the caffeic acid O-methyltransferase COMT consumes $S$-adenosyl- $L$-methionine (SAM), which is a ubiquitous intracellular methyl donor involved in a great number of reactions (Han et al. 2016). Increasing the intracellular SAM supply is considered a generally useful approach for improving the production of natural products (Wang et al. 2007). Methionine adenosyltransferase /SAM synthetase, which catalyzes the formation of SAM from $L$-methionine and ATP, is found in E. coli, baker's yeast, Mycobacterium smegmatis, rat liver, bovine brain, and Saccharomyces cerevisiae (Han et al. 2016). Overexpression of methionine adenosyltransferase may also increase the production of FA.

In this study, we engineered E. coli for efficient FA production. Firstly, we constructed a tal-sam5-comt expression cassette using genes from three different species. The copy number and promoter strength of the FA biosynthetic pathway gene expression cassette were tuned to optimize the pathway. To further increase the yield by supplying the redox cofactor NADPH and methyl donor SAM, five NADPH regeneration enzymes and one SAM producing enzyme were individually overexpressed, resulting an increased FA titer.

\section{Materials and methods}

\section{Bacterial strains, plasmids and chemicals}

E. coli $\mathrm{DH} 10 \mathrm{~B}$ was used for plasmid construction. E. coli JM109(DE3) was used for the expression of heterologous genes and the production of FA. pET vectors 
and pCL1920-T7 were used to express multiple genes. Authentic chemical FA standards, $p$-coumaric acid and caffeic acid were purchased from Yuanye (Shanghai). All restriction enzymes and DNA ligase were purchased from New England Biolabs (Shanghai). One-stepdirected cloning kit was purchased from Novoprotein (Shanghai). The polymerase chain reaction (PCR) primers are synthesized from Sangon Biotech (Shanghai) company (Additional file 1: Table S2). The E. coli strains and plasmids used in this study are listed in Table 1.

\section{Biosynthetic pathway construction and assembly}

Restriction enzyme digestions, transformations, PCR, sodium dodecyl sulfate polyacrylamide gel electrophoresis (SDS-PAGE) analysis, and other molecular biology techniques were carried out by the standard method. The primers used are listed in Additional file 1: Table S2. Cells were grown in Luria-Bertani (LB) medium containing appropriate antibiotics at $37^{\circ} \mathrm{C}$. The working concentration of antibiotics was ampicillin $(100 \mathrm{mg} / \mathrm{L})$, apramycin $(100 \mathrm{mg} / \mathrm{L})$, kanamycin $(50 \mathrm{mg} / \mathrm{L})$, and streptomycin (50 mg/L).

The FA biosynthesis pathway was expressed from the pET21d vector. Tyrosine ammonia-lyase from
Rhodobacter sphaeroides (encoded by tal; GenBank No. CP033447.1) was cloned from pET21d-tal. The $p$-coumaric acid 3-hydroxylase from Saccharothrix espanaensis (encoded by sam5; GenBank No. HE804045.1), and caffeic acid $O$-methyltransferase from Triticum aestivum (encoded by comt; GenBank No. EF413031.1), were codon-optimized and synthesized by GenScript Company and cloned into pET21a to construct pET21a-sam5 and pET21a-comt, respectively. The nucleotide sequences of genes used in this study are listed in Additional file 1: Table S3. The whole pathway was sequentially assembled based on the BioBrick Assembly method using XbaI/SpeI. The XbaI/HindIII excised DNA fragment from pET21a-sam5 was inserted between SpeI/HindIII sites of pET21d-tal to obtain plasmid pET21d-talsam5. Then the XbaI/HindIII excised DNA fragment from pET21a-comt was sequentially inserted between SpeI/HindIII sites of pET21d-tal-sam5 to generate plasmid pET21d-tal-sam5-comt.

To construct the plasmid pHJ345 (pBR322 ori, Apr resistance, T5 promoter) with backbone pBR322-T5, we substituted the T7 promoter in the plasmid pJF25 (Wang et al. 2013) (pBR322 ori, Apr resistance, T7 promoter) with the T5 promoter sequence from $\mathrm{pQE} 30$

Table 1 The E. coli strains and plasmids used in this study

\begin{tabular}{|c|c|c|}
\hline Strain and plasmid & Description & Source \\
\hline JM109(DE3) & $\begin{array}{l}\text { endA1 glnV44 thi-1 relA1 gyrA96 recA1 mcrB }+\triangle(\text { lac-proAB) e14-[F' traD36 proAB }+ \text { lacla } \\
\text { lacZ } \triangle M 15] \text { hsdR17(rK-Mk }+)+\lambda(\mathrm{DE} 3)\end{array}$ & Lab stock \\
\hline $\mathrm{DH} 10 \mathrm{~B}$ & $\begin{array}{l}\text { F-mcrA } \triangle \text { (mrr-hsdRMS-mcrBC) p80lacZ } \triangle M 15 \Delta \text { lacX74 recAlendA1 araD139 (ara, leu)7697 galU } \\
\text { galK-rpsL nupG }\end{array}$ & Lab stock \\
\hline pET21d-tal & pET21d derived, T7 Prom-tal-T7 Term & Lab stock \\
\hline pET21a-sam5 & pET21a derived, T7 Prom-sam5-T7 Term & This study \\
\hline pET21a-comt & pET21a derived, T7 Prom-comt-T7 Term & This study \\
\hline pET21d-tal-sam5 & pBR322 ori, Amp resistance, T7 Prom-tal-sam5-T7 Term & This study \\
\hline pET21d-tal-sam5-comt & pBR322 ori, Amp resistance, T7 Prom-tal-sam5-comt-T7 Term & This study \\
\hline 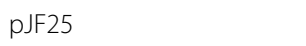 & pBR322 ori, Apr resistance, T7 Promoter & (Wang et al. 2013) \\
\hline $\mathrm{pHJ} 345$ & pBR322 ori, Apr resistance, T5 Promoter & This study \\
\hline pHJ352 & P15a ori, Apr resistance, T7 Promoter & This study \\
\hline pHJ354 - n & P15a ori, Apr resistance, T5 Promoter & This study \\
\hline pBR322-T7-tal-sam5-comt & pBR322 ori, Apr resistance, T7 Prom-tal-sam5-comt-T7 Term & This study \\
\hline pBR322-T5-tal-sam5-comt & pBR322 ori, Apr resistance, T5 Prom-tal-sam5-comt-T7 Term & This study \\
\hline p15a-T7-tal-sam5-comt & P15a ori, Apr resistance, T7 Prom-tal-sam5-comt-T7 Term & This study \\
\hline p15a-T5-tal-sam5-comt & P15a ori, Apr resistance, T5 Prom-tal-sam5-comt-T7 Term & This study \\
\hline pCL1920 & pSC101 ori, spectinomycin resistance, cloning vector & (Lerner and Inouye 1990) \\
\hline pCL1920-T7 & pSC101 ori, spectinomycin resistance, T7 Prom-MCS-T7 Term & Lab stock \\
\hline pCL1920-T7-Ecicd & pSC101 ori, spectinomycin resistance, T7 Prom-Ecicd-T7 Term & This study \\
\hline pCL1920-T7-Ecgnd & pSC101 ori, spectinomycin resistance, T7 Prom-Ecgnd-T7 Term & This study \\
\hline pCL1920-T7-Eczwf & pSC101 ori, spectinomycin resistance, T7 Prom-Eczwf-T7 Term & This study \\
\hline pCL1920-T7-EcpntAB & pSC101 ori, spectinomycin resistance, T7 Prom- EcpntAB-T7 Term & This study \\
\hline pCL1920-T7-CagapN & pSC101 ori, spectinomycin resistance, T7 Prom-CagapN-T7 Term & This study \\
\hline
\end{tabular}


(colE1 ori, Amp resistance, T5 promoter) by fusing a 5.3-kb vector fragment amplified from pJF25 using the primer pair pro-VF/pro-VR and a 110-bp insert fragment amplified from $\mathrm{PQE30}$ using the primer pair pro-T5-in-F/pro-T5-in-R.

Similarly, to construct the plasmid pHJ352 (p15a ori, Apr resistance, T7 promoter) with backbone p15a-T7, we substituted the pBR322 ori in the plasmid pJF25 (pBR322 ori, Apr resistance, T7 promoter) with the $\mathrm{p} 15 \mathrm{~A}$ sequence from pACYC184 (p15A) by fusing a $3.3 \mathrm{~kb}$ vector fragment amplified from pJF25 using the primer pair ori-VF/ori-VR and a 913 bp insert fragment amplified from pQE30 using the primer pair ori-p15a-inF/ori-p15a-inR.

The plasmid pHJ354(p15a ori, Apr resistance, T5 promoter) with backbone p15a-T7 was constructed by fusing a 4.3-kb vector fragment amplified from $\mathrm{pHJ} 352$ using the primer pair pro-VF/pro-VR and a 110-bp insert fragment amplified from $\mathrm{pQE30}$ using the primer pair ori-p15a-inF/ori-p15a-inR.

FA biosynthesis pathways with different backbones, pBR322-T7-tal-sam5-comt, pBR322-T5-tal-sam5-comt, p15a-T7-tal-sam5-comt and p15a-T5-tal-sam5-comt were constructed by T4 ligase using XbaI/HindIII excised DNA fragment from pET21d-tal-sam5-comt as insert fragment, and XbaI/HindIII excised DNA fragments from pJF25, pHJ345, pHJ352, pHJ354 as vector fragments, respectively.

We modified low-copy plasmid pCL1920 (G.Lerner and Inouye 1990) to get pCL1920-T7 to co-express NADPH regeneration system with FA biosynthetic pathway. pCL1920-T7 is constructed by infusion method using two PCR products: using pCL1920 as template and primers pCL1920VF/pCL1920VR to obtain a $4.0 \mathrm{~kb}$ DNA fragment as vector fragment, and using pET28a as template and primers T7 operator F/ T7 operator R to obtain a 1.9-kb DNA fragment as insert fragment.

The genes $z w f$, gnd, icd and pntAB were PCR amplified from the genomic DNA of E. coli MG1655 and cloned into the pET28a vector. Subsequently the digested fragments were inserted into pCL1920-T7 to generate pCL1920-T7- $z w f$, pCL1920-T7-gnd, pCL1920-T7$i c d$, pCL1920-T7-pntAB, respectively. The gapN gene from Clostridium. acetobutylicum ATCC 824 (Genbank No. AE001437.1) was fused with the pCL1920-T7 backbone to generate pCL1920-T7-gapN. The metK gene from Streptomyces spectabilis (Genbank No. WP_144002349.1) was codon-optimized and synthesized by Genscript Company. The synthetic fragment was cloned into pET21a, and subcloned into pCL1920-T7 using XbaI/HindIII to generate pCL1920-T7-metK. All constructs were verified by Sanger sequencing (Sangon Biotech).

\section{Flask fermentation}

The clones transformed with the indicated plasmid combinations were picked from plates and grown in LB medium at $37{ }^{\circ} \mathrm{C}$ and $250 \mathrm{rpm}$ for $12 \mathrm{~h}$. On the next day, $500 \mu \mathrm{L}$ of the resulting seed culture was used to inoculate $10 \mathrm{~mL}$ of M9Y medium supplemented with $2 \%(\mathrm{v} / \mathrm{v})$ glycerol and $1 \mathrm{~g} / \mathrm{L} L$-tyrosine based on a previous publication (Ni et al. 2015), in which higher specific titer was obtained by using this medium compared with LB medium or M9 minimal medium, and glycerol was suggested as more suitable carbon source than glucose. M9Y medium is modified M9 minimal salt medium containing $1 \mathrm{~g} \mathrm{NH}_{4} \mathrm{Cl}, 6 \mathrm{~g} \mathrm{Na}_{2} \mathrm{HPO}_{4}, 3 \mathrm{~g} \mathrm{KH}_{2} \mathrm{PO}_{4}, 0.5 \mathrm{~g} \mathrm{NaCl}$, $2 \mathrm{mmol} \mathrm{MgSO}_{4}, 0.1 \mathrm{mmol} \mathrm{CaCl}_{2}, 0.5 \mathrm{~g}$ yeast extract and $1 \mathrm{~mL}$ trace elements $\left(0.03 \mathrm{~g} / \mathrm{L} \mathrm{H}_{3} \mathrm{BO}_{3}, 1 \mathrm{~g} / \mathrm{L}\right.$ thiamine, $0.94 \mathrm{~g} / \mathrm{L} \mathrm{ZnCl} 2,0.5 \mathrm{~g} / \mathrm{L} \mathrm{CoCl}_{2}, 0.38 \mathrm{~g} / \mathrm{L} \mathrm{CuCl}, 1.6 \mathrm{~g} / \mathrm{L}$ $\mathrm{MnCl}_{2}$ and $3.6 \mathrm{~g} / \mathrm{L} \mathrm{FeCl}_{2}$ ) per liter. IPTG was added to the cultures to a final concentration of $0.1 \mathrm{mM}$, and cultures were transferred to a shaker at $28{ }^{\circ} \mathrm{C}, 250 \mathrm{rpm}$ for 3-5 days. Samples were collected at intervals of $24 \mathrm{~h}$ when needed to monitor the $\mathrm{OD}_{600}$ and product concentration by HPLC (high-performance liquid chromatography). All the experiments were conducted in triplicate.

\section{Analytical methods}

Cell growth was monitored by measuring the absorbance at $600 \mathrm{~nm}\left(\mathrm{OD}_{600} \mathrm{~nm}\right)$ with an UV-1200 spectrophotometer (APADA).

The products FA, $p$-coumaric acid, and caffeic acid in culture were extracted twice by an equivalent volume of ethyl acetate. The supernatant was collected and evaporated to dryness and dissolved in an equal volume methanol. The samples were analyzed by high-performance liquid chromatography on an Ultimate 3000 system (Thermo Scientific, USA) using a C18 column (Silgreen HPLC column $250 \times 4.6 \mathrm{~mm}$; particle size, $5 \mu \mathrm{m}$ ) and a DAD detector at $310 \mathrm{~nm}$. Compounds were separated by water (containing $0.1 \%$ acetic acid, mobile phase $\mathrm{B}$ ) and methanol (containing $0.1 \%$ acetic acid, mobile phase C) at a flow rate of $1.0 \mathrm{~mL} / \mathrm{min}$ and column temperature $30{ }^{\circ} \mathrm{C}$ under the following conditions: $80 \% \mathrm{~B}+20 \% \mathrm{C}$ in $0-2 \min , 50 \% \mathrm{~B}+50 \% \mathrm{C}$ in $15 \mathrm{~min}, 80 \% \mathrm{~B}+20 \% \mathrm{C}$ in $17 \mathrm{~min}, 80 \% \mathrm{~B}+20 \% \mathrm{C}$ in $20 \mathrm{~min}$. The retention time of FA, $p$-coumaric acid, and caffeic acid were $17.318 \mathrm{~min}$, $16.5 \mathrm{~min}$, and $12.99 \mathrm{~min}$, respectively. The compounds were quantified based on calibration curves of various concentrations of standards using peak area.

\section{Accession numbers}

The GenBank accession numbers for the nucleotide sequence of the codon-optimized tal, sam5, comt and metK genes are MW403919, MW403920, MW403921 and MW403922, respectively. 


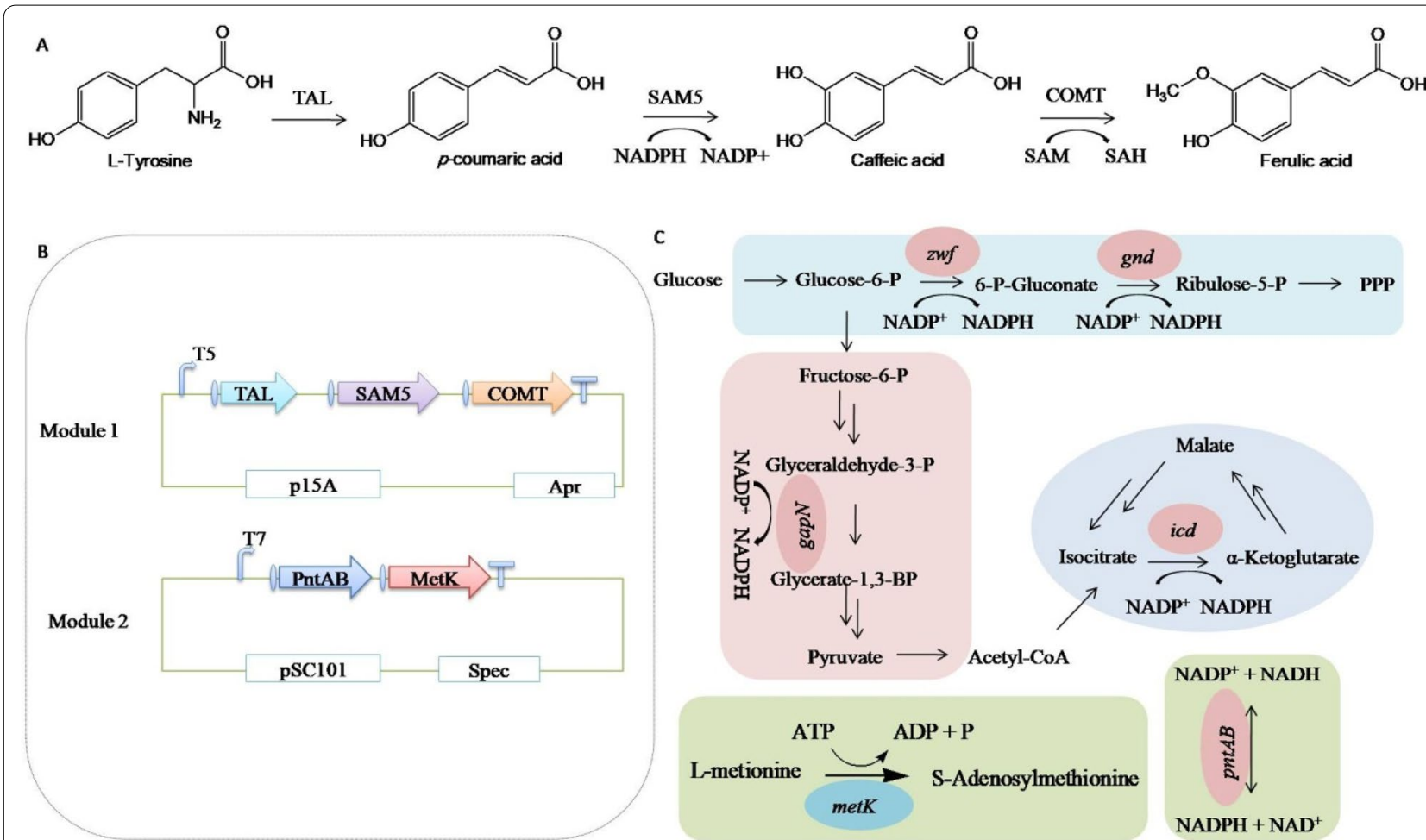

Fig.1 Construction of a heterologous FA biosynthesis pathway in E. coli (A). Tyrosine ammonia-lyase (TAL) catalyzes the non-oxidative deamination of the primary amino acid tyrosine into $p$-coumaric acid, which is converted into caffeic acid through hydroxylation at the 3-position of the benzyl ring by the $p$-coumarate 3-hydroxylase (SAM5). Finally, FA is biosynthesized from caffeic acid by the enzyme caffeate O-methyltransferase (COMT). Schematic representation of the FA biosynthesis pathway (B). NADPH and SAM regenerating enzymes used to increase FA production (C)

\section{Results}

Construction of a heterologous FA biosynthetic pathway

To construct a heterologous FA biosynthesis pathway (Fig. 1A), the tal, sam5 and comt genes, respectively, encoding tyrosine ammonia-lyase, $p$-coumarate 3-hydroxylase, and caffeic $O$-methyltransferase from Rhodobacter sphaeroides, Saccharothrix espanaensis, and Triticum aestivum, respectively, were codon-optimized, synthesized, and assembled into pET21d/a or plasmid as a single operon under the control of the strong T7 promoter, resulting pBR322-T7-tal-sam5-comt. The construct was introduced to E. coli BL21(DE3) and JM109(DE3), and the recombinant E. coli strains were fermented in Terrific Broth (TB) medium with additional $1 \mathrm{~g} / \mathrm{L} L$-tyrosine and $2 \%(\mathrm{v} / \mathrm{v})$ glycerol at $28{ }^{\circ} \mathrm{C}$ and $250 \mathrm{rpm}$ for 3 days. FA, caffeic acid and $p$-coumaric acid were detected via HPLC analysis (Fig. 2A), but the final product $\mathrm{FA}$ was obtained with little $p$-coumaric acid residual in the fermentation broth. The data showed that the strain based on the E. coli JM109(DE3) chassis produced significantly more FA than the strain based on BL21(DE3) (60 vs. $14 \mathrm{mg} / \mathrm{L}$, Fig. 2B). Thus, E. coli JM109(DE3) was chosen as the host cell for FA production in further experiments.
The protein expression levels of tal, sam5 and comt were very high under the control of the T7 promoter at all tested temperatures $\left(37,28,22^{\circ} \mathrm{C}\right)$, but the protein solubility was improved at lower temperatures (Additional file 1: Figure $\mathrm{S} 1 \mathrm{~A}-\mathrm{C}$ ). In order to alleviate the expression burden, we exchanged the very strong T7 promoter with the less extreme T5 promoter. The expression level of each gene was lower under the control of the T5 promoter, but the solubility at $28^{\circ} \mathrm{C}$ was improved compared to the T7 promoter (Additional file 1: Figure S1D). The new expression cassette containing tal, sam 5 and comt under the control of the T5 promoter was introduced into E. coli JM109 (DE3), resulting in the strain T5FA. Subsequently, the recombinant $E$. coli T5FA was fermented in M9Y medium supplemented with $2 \%(\mathrm{v} / \mathrm{v})$ glycerol and $1 \mathrm{~g} / \mathrm{L} L$-tyrosine, as described in a vanillin biosynthesis study ( $\mathrm{Ni}$ et al. 2015). The fermentation profile showed that the titers of FA and caffeic acid increased quickly in the first 2 days, and, respectively, peaked at 130 and $33 \mathrm{mg} / \mathrm{L}$ after $48 \mathrm{~h}$. By contrast, the titer of $p$-coumaric acid decreased in the first two days and was practically undetectable after $48 \mathrm{~h}$ (Fig. 2C). 

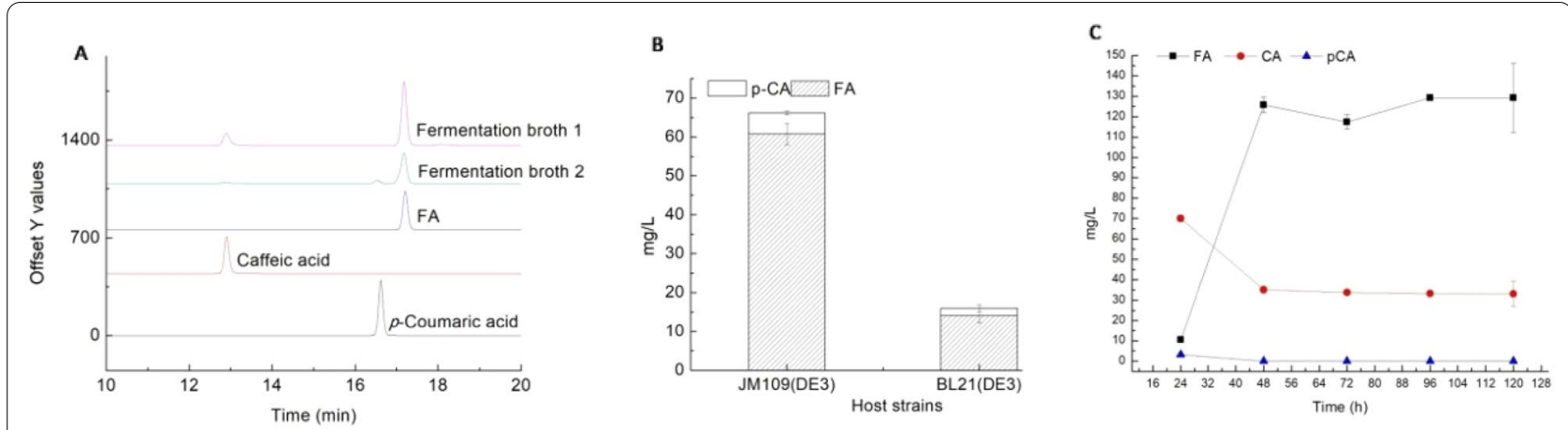

Fig.2 Heterologous biosynthesis of FA in E. coli. A HPLC profiles of the fermentation products and the FA, p-coumaric acid and caffeic acid standards. B FA production by strains based on E. coli JM109(DE3) and BL21(DE3) carrying the plasmid pBR322-T7-tal-sam5-comt. The fermentation was carried out in terrific broth (TB) medium with the addition of $1 \mathrm{~g} / \mathrm{L} L$-tyrosine and $2 \%(\mathrm{v} / \mathrm{v})$ glycerol at $28^{\circ} \mathrm{C}$ and $250 \mathrm{rpm}$ for 3 days. $p$ - CA p-coumaric acid; FA trans-ferulic acid. C Time course of FA production from L-tyrosine by E. coli JM109(DE3) T5FA in M9Y medium supplemented with $2 \%(\mathrm{~V} / \mathrm{V})$ glycerol and $1 \mathrm{~g} / \mathrm{L} L$-tyrosine at $28^{\circ} \mathrm{C}$ and $250 \mathrm{rpm}$ for 5 days

\section{Pathway optimization by copy number and promoter strength tuning}

As mentioned above, tuning the expression levels of pathway enzymes could impact greatly on the final production, here, we replaced the medium copy number replicon pBR322 of FA biosynthesis pathway with the lower copy number replicon $\mathrm{p} 15 \mathrm{a}$. In order to keep the same antibiotic resistance, pET21d-tal-sam5-comt was further constructed to pBR322-T7-tal-sam5-comt, and no significant FA yields differences were observed (data not shown). Combined with promoter T7 or T5, FA biosynthesis pathway in plasmid backbones pBR322-T5, pBR322-T7, p15a-T5 and p15a-T7 (Fig. 1B), with different expression levels of pathway enzymes were obtained. Flask fermentation was carried out at the same condition as previous mentioned and the result showed that strain p15aT5 with FA biosynthesis pathway in plasmid p15a-T5-tal-sam5-comt works best (Fig. 3). FA production of strain p15aT5 reached $180 \mathrm{mg} / \mathrm{L}$ which is significantly higher than other strains. And there was little caffeic acid residual $(\sim 5 \mathrm{mg} / \mathrm{L})$ in the fermentation, compared with strain T5FA, in which FA and caffeic acid production was $130 \mathrm{mg} / \mathrm{L}$ and $33 \mathrm{mg} / \mathrm{L}$, respectively.

The fermentation profiles of most strains showed that the FA production peaked after 2 or 3 days, the strains coexpressing NADPH regenerating enzymes peaked later, after 4 days. We detected the production in day 3 and day 5 , and showed the data of day 5 here.

\section{Improving FA production by NADPH regeneration enzyme Further improvement of FA production by increased NADPH regeneration}

The transformation of $p$-coumaric acid into caffeic acid in the FA biosynthesis pathway is catalyzed by NADPH consuming $p$-coumarate 3-hydroxylase (SAM5) (Fig. 1A).To increase NADPH availability for

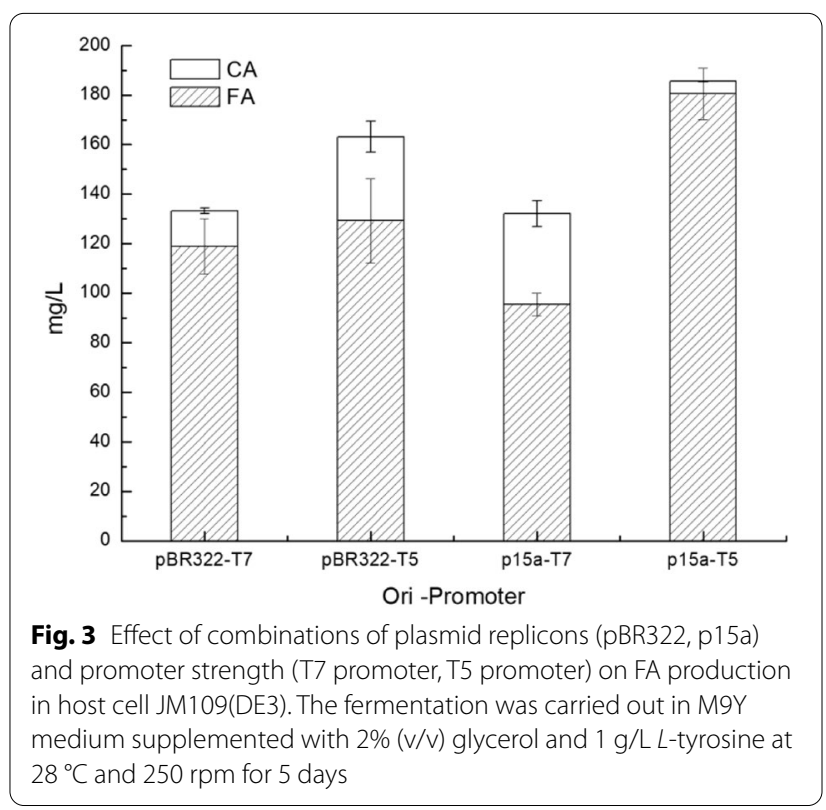

FA production, genes encoding NADPH regeneration enzymes (Fig. 1C), including $z w f$, gnd, icd, and pntAB from $E$. coli MG1655, as well as gap $\mathrm{N}$ form Clostridium acetobutylicum ATCC 824 (GenBank No. AE001437.1) were individually cloned into the low-copy-number vector pCL1920-T7 ( 5 copies per cell) and introduced into JM109(DE3) together with the FA biosynthesis pathway.

The strain T5FA + pntAB, co-expressing the pntAB genes with the FA biosynthesis pathway, produced $192 \pm 9.6 \mathrm{mg} / \mathrm{L}$ FA with $30 \pm 10.0 \mathrm{mg} / \mathrm{L}$ residual caffeic acid, which was a significant improvement compared with the $140 \pm 7.7 \mathrm{mg} / \mathrm{L} \mathrm{FA}$ and $30 \pm 14.8 \mathrm{mg} / \mathrm{L}$ residual caffeic acid of the control strain T5FA + pCL1920-T7. 
Interestingly, FA production of the control strain $E$. coli T5FA + pCL1920-T7 (140 mg/L FA and $30 \mathrm{mg} / \mathrm{L}$ caffeic acid, Fig. 4) was marginally higher than that of $E$. coli T5FA expressing only the FA biosynthesis pathway (130 mg/L FA and $33 \mathrm{mg} / \mathrm{L}$ caffeic acid, Fig. 2c). Strains expressing the other candidate genes ( $i c d, z w f$, gnd, gapN) exhibited intermediate FA titers, which were higher than that of strain T5FA, but lower than that of the control strain co-transfected with the empty vector PCL1920-T7.

\section{Improving FA production by non-native SAM synthetase}

To increase SAM availability for FA production, the nonnative metK from Streptomyces spectabilis (Genbank No. WP_144002349.1) was cloned and fused downstream of pntAB, forming a bicistronic structure with pntAB under the control of T7 promoter on the low-copy vector pCL1920-T7 ( $\sim 5$ copies per cell), and introduced into JM109(DE3) together with FA biosynthesis pathway p15a-T5-tal-sam5-comt. The strain p15aT5 + pntAB + metK co-expressing pntAB gene and $m e t K$ gene with FA biosynthesis pathway p15aT5-tal-sam5-comt could

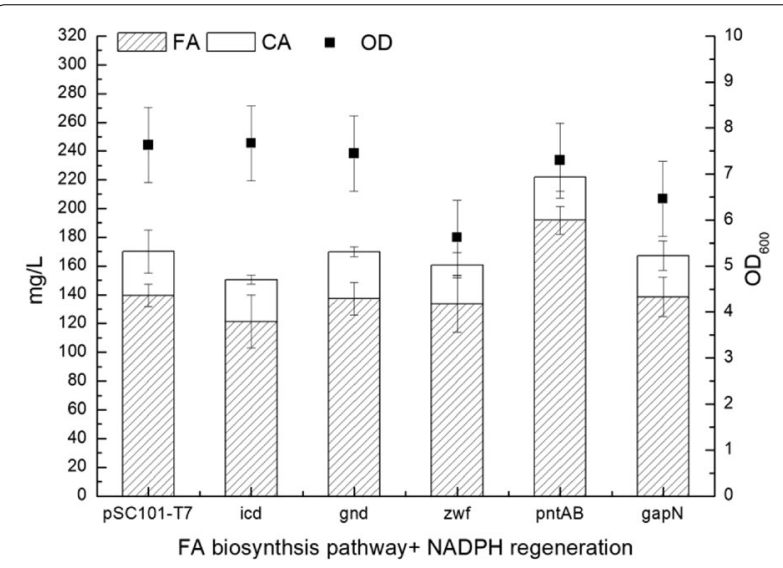

Fig. 4 Comparison of FA biosynthesis strains co-expressed with NADPH regenerating enzymes. pSC101-T7 refers to FA biosynthesis pathway plasmid pBR322-T5-tal-sam5-comt co-expressed with plasmid PCL1920-T7 in host cell JM109(DE3); icd refers to FA biosynthesis pathway plasmid pBR322-T5-tal-sam5-comt co-expressed with plasmid PCL1920-T7-Ecicd in host cell JM109(DE3), gnd refers to FA biosynthesis pathway plasmid pBR322-T5-tal-sam5-comt co-expressed with plasmid pCL1920-T7- Ecgnd in host cell JM109(DE3), zwf refers to FA biosynthesis pathway plasmid pBR322-T5-tal-sam5-comt co-expressed with plasmid pCL1920-T7-Eczwf in host cell JM109(DE3), pntAB refers to FA biosynthesis pathway plasmid pBR322-T5-tal-sam5-comt co-expressed with plasmid pCL1920-T7- EcpntAB in host cell JM109(DE3), gapN refers to FA biosynthesis pathway plasmid pBR322-T5-tal-sam5-comt Co-expressed with plasmid pCL1920-T7-CagapN in host cell JM109(DE3). The fermentation was carried out in M9Y medium supplemented with $2 \%(\mathrm{~V} / \mathrm{V}) \mathrm{glycerol}$ and $1 \mathrm{~g} / \mathrm{L} \mathrm{L}$-tyrosine at $28^{\circ} \mathrm{C}$ and $250 \mathrm{rpm}$ for 5 days increase the production significantly compared with the control strain p15aT5 + pCL1920-T7 (Fig. 5), the production was $212.5 \pm 11.4 \mathrm{mg} / \mathrm{L} \mathrm{FA}$ and $11.9 \pm 0.5 \mathrm{mg} / \mathrm{L}$ caffeic acid vs $178.3 \pm 1.8 \mathrm{mg} / \mathrm{L}$ FA and $15.4 \pm 0.6 \mathrm{mg} / \mathrm{L}$; but no significant difference with strain p15aT5 + pntAB $(207.3 \pm 2.9 \mathrm{mg} / \mathrm{L}$ FA and $13.0 \pm 0.7 \mathrm{mg} / \mathrm{L}$ caffeic acid $)$. The addition of $L$-methionine during fermentation process decreased FA production surprisingly (Additional file 1: Table S4).

\section{Discussion}

Construction of a heterologous FA biosynthesis pathway

The exogenous genes tal (Genbank No. CP033447.1), sam5 (Genbank No. HE804045.1) and comt (Genbank No. EF413031.1) comprising FA biosynthesis pathway were selected based on previous studies (Berner et al. 2006; Ma and Xu 2008; Watts et al. 2004). Jendresen et al. reported that highly efficient tyrosine ammonialyases (TALs) from diverse origins enable enhanced production of aromatic compounds in bacteria and Saccharomyces cerevisiae (Jendresen et al. 2015). The tal gene (Genbank No. CP033447.1) performed well in

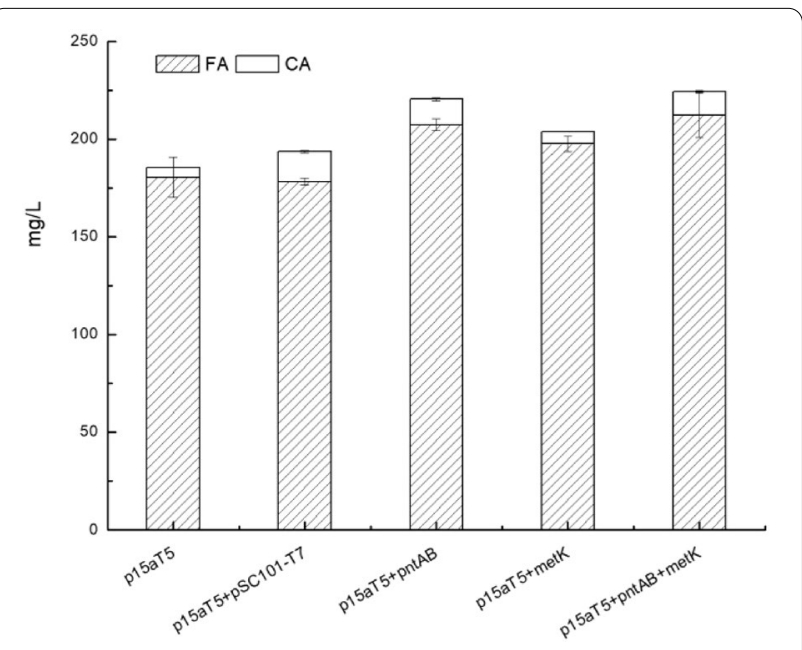

Fig. 5 Effect of combination of NADPH regenerating enzyme gnt $A B$ with $S$-adenosyl-L-methionine synthetase metK in FA biosynthesis strain. p15a-T5 refers to FA biosynthesis pathway plasmid p15a-T5-tal-sam5-comt in host cell JM109(DE3); p15a-T5 + pSC101-T7 refers to FA biosynthesis pathway plasmid p15a-T5-tal-sam5-comt co-expressed with plasmid pCL1920-T7 in host cell JM109(DE3); p15a-T5 + pntAB refers to FA biosynthesis pathway plasmid p15a-T5-tal-sam5-comt co-expressed with plasmid pCL1920-pntAB in host cell JM109(DE3); p15a-T5 + metK refers to FA biosynthesis pathway plasmid p15a-T5-tal-sam5-comt co-expressed with plasmid pCL1920-metK in host cell JM109(DE3); p15a-T5 + pntAB + metK refers to FA biosynthesis pathway plasmid p15a-T5-tal-sam5-comt co-expressed with plasmid pCL1920-pntAB-metK in host cell JM109(DE3); The fermentation was carried out in M9Y medium supplemented with $2 \%(\mathrm{~V} / \mathrm{V})$ glycerol and $1 \mathrm{~g} / \mathrm{L} \mathrm{L}$-tyrosine at $28^{\circ} \mathrm{C}$ and $250 \mathrm{rpm}$ for 5 days 
our previous study on the de novo biosynthesis of resveratrol in E. coli (Wang et al. 2014), and the expression of the FA biosynthesis pathway using BL21(DE3) as the host cell resulted in $p$-coumaric acid accumulation (Fig. 2A). Rodrigues et al. compared $p$-coumarate 3-hydroxylase from Saccharothrix espanaensis (sam5) and Rhodopseudomonas palustris (CYP199A2) for the conversion of $p$-coumaric acid produced from tyrosine into caffeic acid in E. coli. The CYP199A2 enzyme was more active, but it requires two redox partners, which may complicate its heterologous expression (Haslinger and Prather 2020; Rodrigues et al. 2015a). COMT from A. thaliana (GenBank No. AY062837) was previously used to catalyze caffeic acid to FA in several reports (Choi et al. 2011; Heo et al. 2017). Here we tested COMT from wheat (Triticum aestivum L. cv. H4564, GenBank No. EF413031.1); this enzyme was named $\mathrm{TaCM}$ in original literature, and was proposed to methylate phenol substrates containing aldehyde, flavonoid and CoA moieties. Thus it may have a broad substrate preferences, and can not only involves in converting caffeic acid to ferulic acid and 5-hydroxyferulic acid to sinapic acid, but also in the conversion of caffeoylCoA to feruloyl-CoA, and 5-hydroxyferuloyl-CoA to sinapoyl-CoA (Ma and Xu 2008). Notably the protein sequence of $\mathrm{TaCM}$ has $100 \%$ identity with TaOMT2 (Genbank No. DQ223971), which showed the highest activity specifically to tricetin in substrate preference test against a number of phenolic compounds, and was proposed to accept caffeic acid and 5-hydoxy-ferulic acid as substrates (Zhou et al. 2006, 2009). Here, we used $\mathrm{TaCM}$ in the FA biosynthesis pathway and confirmed its ability to convert caffeic acid into FA. To our best knowledge, this is the first report of the caffeic acid $\mathrm{O}$-methyltransferase activity of TaCM/TaOMT2.

E. coli BL21(DE3) is generally a more effective expression host than JM109(DE3), and the latter is a high acetate producer (Shiloach et al. 1996), while acetate accumulation can reduce the growth rate and recombinant protein synthesis (Noronha et al. 2000). In a study by Kang et al. E. coli C41(DE3) [mutant derivative of BL21(DE3)] was engineered as a tyrosine over-producing chassis for the biosynthesis of phenylpropanoic acid (Kang et al. 2012). Similarly, Huang et al. used $E$. coli BW25113 (mutant derivative of $E$. coli $\mathrm{K}-12$ ) for the biosynthesis of caffeic acid (Huang et al. 2013; Lin and Yan 2012). In this study, the heterologous biosynthetic pathway under the control of the strong T7 promoter was tested in E. coli JM109(DE3) and BL21(DE3), and the results showed that it could convert $L$-tyrosine into FA, with a small amount of residual caffeic or $p$-coumaric acid (Fig. 2a). Interestingly, the FA yields were higher when using JM109(DE3) as the host strain than with BL21(DE3), so JM109(DE3) was selected as heterologous expression host cell for further experiments.

To increase the FA titer, we firstly attempted to improve soluble protein expression using the T7 promoter by decreasing the temperature form $37-22{ }^{\circ} \mathrm{C}$. SDS-PAGE analysis showed that TAL and SAM5 were mostly expressed in the form of inclusion bodies, and their solubility was slightly improved at the lower temperature (Additional file 1: Figure S1A-C). We next replaced the strong $\mathrm{T} 7$ promoter with the weaker T5 promoter, and protein expression was changed greatly. Although the total protein expression level decreased, the solubility was improved, especially for TAL and SAM5. Based on this, we replaced the T7 promoter in the FA expression cassette with the T5 promoter, and the FA titer in M9Y medium increased to $130 \mathrm{mg} / \mathrm{L}$ (Fig. 2C). This result suggests that the FA yield could be optimized by tuning the gene expression to an appropriate level.

\section{Pathway optimization by copy number and promoter strength tuning}

Using different combinations of plasmid replicons and promoter strengths is convenient to tune the expression levels of pathway genes. Modulating diverse expression level of up- and downstream pathways of taxadiene synthesis resulted in a remarkable 15,000-fold increase of taxadiene titer in an engineered $E$. coli strain. Here our results of different plasmid replicon ( $\mathrm{p} 15 \mathrm{a}$, about 10 copies; pBR322, about 20 copies) and promoter strength (T7, the relative strength is 4.97 ; $\mathrm{T} 5$, the relative strength is 2) combinations showed strain p15a-T5 yield the highest FA titer, $180.5 \pm 10 \mathrm{mg} / \mathrm{L} \mathrm{FA}, 5 \pm 0.1 \mathrm{mg} / \mathrm{L}$ caffeic acid $\mathrm{mg} / \mathrm{L}$. The following two were strain pBR322-T5 and pBR322-T7, and the production of strain p15a-T7 was the lowest. The order of the corresponding replicon copies and promoter strength combinations is $10-2>20-2>$ $20-4.97>10-4.97$, FA titer decline in turn. The numbers refers to replicon copies and promoter strength in Ajikumar's work (Ajikumar et al. 2010). More work is needed for even refined modulation, like screening for combinations of promoters and terminators, modular controlling via different promoters, using RBS libraries that covered a broad range of translational initiation rates, and so on (Zhang and Hong 2020), and it is becoming a universal approach to achieve high yield for heterologous biosynthesis.

It is worth noting that, production of strain pBR322T7 $(118.8 \pm 11 \mathrm{mg} / \mathrm{L}$ FA, $14.4 \pm 1.0 \mathrm{mg} / \mathrm{L}$ caffeic acid $)$ is higher than that previous fermentation carried in TB, indicating culture medium composition may affect the yield. 
Improving FA production by NADPH regeneration enzyme In the FA biosynthetic pathway, $p$-coumarate 3-hydroxylase (SAM5) consumes NADPH. Thus, the overexpression of heterologous pathway can greatly affect the intracellular redox homeostasis. The overexpression or introduction of a heterologous cofactor regeneration system is an important strategy for engineering redox homeostasis, and this approach has been successfully used for the biosynthesis of many NAD(P)-dependent products (Liu et al. 2018; Zhao et al. 2017). Here, we tested five NADPH regenerating enzymes that were proved to increase the NADPH/NADP ${ }^{+}$ratio in E. coli (Huang et al. 2017), covering almost all NADPH regeneration systems for E. coli. The glucose-6-phosphate dehydrogenase ZWF and 6-phosphogluconate dehydrogenase GND are involved in the PP Pathway. Similarly, the glyceraldehyde3-phosphate dehydrogenase GapN from Clostridium acetobutylicum ATCC 824 is involved in the glycolysis pathway, and the isocitrate dehydrogenase ICD participates in the TCA cycle. By contrast, the transhydrogenase Pnt $A B$ catalyzes the transfer of reducing power from $\mathrm{NADH}$ to $\mathrm{NADP}^{+}$(Fig. 1C). The strain co-expressing the pntAB genes with the FA biosynthesis pathway (T5FA + pntAB) exhibited a significant increase of FA production $(191.9 \pm 9.6 \mathrm{mg} / \mathrm{L}$; Fig. 4). However, the other NADPH regenerating enzymes showed no effect on the FA titer. Similarly, pntAB overexpression to mitigate the redox imbalance significantly increased the product titer and yield in the biosynthesis of shikimic and glycolic acid in E. coli (Cabulong et al. 2019; Cui et al. 2014), as well as $L$-lysine, acetic and succinic acid in Corynebacterium glutamicum (Kabus et al. 2007; Yamauchi et al. 2014). Sauer et al. found that PntAB produced $35-45 \%$ of the NADPH consumed during the exponential batch growth phase on glucose, while the pentose phosphate pathway and isocitrate dehydrogenase contributed $35-45 \%$ and $20-25 \%$, respectively (Sauer et al. 2004). Based on this, PntAB contributed the most among the five tested enzymes, which may explain why the strain co-expressing the pntAB genes with the FA biosynthesis pathway showed a significant increase of the product titer, which was not observed with the other tested genes. However, glycerol was used as the carbon source in this work, and the effect may be different if glucose is used in the case of the other NADPH regenerating enzymes, especially $z w f$ and gnd, which are involved in the pentose phosphate pathway.

We noted that the FA production of the control strain E. coli T5FA + pCL1920-T7 (140 mg/L FA and $30 \mathrm{mg} / \mathrm{L}$ caffeic acid, Fig. 4) was slightly higher than that of $E$. coli T5FA, which carries only FA biosynthesis pathway plas$\operatorname{mid}(130 \mathrm{mg} / \mathrm{L}$ FA and $33 \mathrm{mg} / \mathrm{L}$ caffeic acid, Fig. 2C). The product titers of the strains expressing the other candidate genes (icd, $z w f$, gnd, gapN) were also lower than that of the control strain. As an amino glycoside antibiotic, spectinomycin binds to the bacterial $30 \mathrm{~S}$ ribosomal subunit and blocks protein synthesis. In the strain carrying the pCL1920 plasmid, the protein synthesis maybe affected due to the addition of spectinomycin to the culture medium, which may reduce the burden of protein synthesis, and improve the yield of FA. Our results also showed that the strain co-transfected with a plasmid carrying a spectinomycin resistance gene exhibited better growth and FA production than the strains without this plasmid.

\section{Improving FA production by non-native SAM synthetase}

The transformation of caffeic acid into FA consumes $S$-adenosylmethionine (SAM) at the same time (Fig. 1). Similar as redox cofactor NADPH, SAM is a ubiquitous intracellular methyl or methylate donor, previous work reported improving heterologous polyketide production in $E$. coli by overexpression of an non-native $S$-adenosylmethionine synthetase (SsmetK) gene (Wang et al. 2007). Increased intracellular SAM availability is beneficial to increase FA production in theory. Endogenous $S$-adenosylmethionine synthetase gene $m e t K$ (SAMs, catalyzing the reaction of ATP and $L$-Methionine to form SAM, Fig. $1 C$ ) in $E$. coli is inhibited by $L$-methionine (Zocchi et al. 2003), for this reason, we chose the non-native metK from Streptomyces spectabilis and tested in our heterologous biosynthesis pathway.

The FA production of strain $\mathrm{p} 15 \mathrm{aT} 5+\operatorname{met} K$, strain p15aT5 + gntAB + metK, and strain p15aT5 + gnt $A B$ is significantly higher than the control strain p15aT5 + pCL1920-T7, the highest one is p15aT5 + gnt $A B+$ metK, producing $212.5 \pm 11.4 \mathrm{mg} / \mathrm{L}$ FA with $11.9 \pm 0.5 \mathrm{mg} / \mathrm{L}$ caffeic acid residue. Caffeic acid accumulation was lowest in strain p15aT5 + metK among these strains, which may attribute to supplement of SAM. Compared with strain T5FA + pntAB $(191.9 \pm 9.6 \mathrm{mg} / \mathrm{L} \mathrm{FA}$ and $30 \pm 10 \mathrm{mg} / \mathrm{L}$ caffeic acid $)$, strain p15aT5 + gntAB $(207.4 \pm 2.9 \mathrm{mg} / \mathrm{L}$ FA and $13 \pm 0.7 \mathrm{mg} / \mathrm{L}$ caffeic acid) produced more FA and left less caffeic acid residual, which is consistent with the expression level optimization objective. While there is no significant difference of FA production between these three strains (Fig. 5), which means that the effect of gene met $K$ on FA production is almost equal with gene $p n t A B$. The strain p15aT5 + pntAB + metK showed no increase of the FA production further, not as expected a possible superimposed promotion effect. We also tried adding $L$-methionine in culture medium directly, but resulted in decreased FA production (Additional file 1: Table S4). Kunjapur et al. reported deletion of metJ coupled with expression of feedback-desensitized variants of met $^{*}$ and $c y s E^{*}$, genes involved in methionine 
biosynthesis, improved de novo vanillate titers by $33 \%$ in an engineered $E$. coli K-12 MG1655 strain RARE that serves as a platform for aromatic aldehyde biosynthesis. While overexpression of $m t n$ and $l u x S$, genes involved in $S$-adenosylhomocysteine (SAH) recycling improved de novo vanillate titers by $25 \%$, it is supposed to work by the mechanism of increasing SAM availability, since SAH is a potent inhibiter of SAM-dependent methyltransferases. And vanillate production improved further upon supplementation with methionine (Kunjapur et al. 2016). So, it seems that methylation improvement in biosynthetic pathway in $E$. coli could be achieved by methods of improving SAM availability like introducing heterologous methionine adenosyltransferase (SsmetK), engineering methionine biosynthesis related genes (met), metA* and $\left.c y s E^{*}\right)$ and SAH recycling related genes ( $m t n$ and $l u x S$ ). As pointed out in Kunjapur et al.s work, adding methionine to E. coli decreases flux in the Met and SAM biosynthetic pathway due to feedback inhibition of MetA. The effect of supplying methionine exogenously may vary from case to case. SAM availability in FA biosynthesis may improve and gain increased FA titer by combining all these strategies in future work. So, the highest FA titer in our work is $212 \mathrm{mg} / \mathrm{L}$, although the FA titer of Kang et al 2012 is $196 \mathrm{mg} / \mathrm{L}$ in $36 \mathrm{~h}$, and our data are $212 \mathrm{mg} / \mathrm{L}$ in 5 days, it seems that the productivity of the former is better, but the carbon supplement of the former is much higher and the host cell is BL21(DE3) (Additional file 1: Table S1). In parallel with our work, Rodrigues et al. reported even higher FA production $(257 \mathrm{mg} / \mathrm{L})$ in their work of curcuminoids biosynthesis in E. coli BL21(DE3) recently. They constructed TAL from Rhodotorula glutinis and C3H/SAM5 from S. espanaensis in pCDF-duet backbone (CloDF13 ori, PT7lac), and COMT from A. thaliana in pRSF-duet backbone (RSF1030 ori, PT7lac) simultaneously. Except for different source of TAL and COMT with our work, BL21(DE3) was chosen as host cell and two plasmids were used to construct FA biosynthesis pathway in their work (Rodrigues et al. 2020). The genes they chose were reported as most the efficient ones, while gene tal and gene comt were not the same version as those used in our work, and FA biosynthesis pathway was constructed in a combination of two plasmids, pCDF-duet_TAL (20-40 copies, PT7lac) and pRSF-duet_C3H_COMT (10 copies, PT7lac). Both the plasmid copy number and promoter strength are higher than the ones used in our work, but reached a balance in their work, although introduction of multiple plasmids is inconvenient for follow-up operations, indicating FA production could be further improved by combined application of two strategies.

In this study, we constructed a heterologous FA biosynthesis pathway by introducing genes from three different species, and the caffeic acid $O$-methyltransferase activity of $\mathrm{TaCM}$ was shown for the first time. FA production was tested in two common E. coli strains, and using JM109(DE3) as the host strain resulted in a higher FA yield than using BL21(DE3). Tuning down the promoter strength of the expression cassette significantly increased the final FA titer, so we continued to optimize the pathway expressing level using combinations of different replicons and promoter strengths, which further increased FA production. To further increase the production, the endogenous NADPH regeneration genes $p n t A B$ and the heterologous SAM formation enzyme SsmetK were used to improve the supply of the key cofactors NADPH and SAM, which greatly increased the FA titer from 130 to $212 \mathrm{mg} / \mathrm{L}$. Further studies are needed to test for synergistic effects of simultaneous NADPH and SAM supplementation, and relevant methods such as increasing methionine biosynthesis and SAH recycling to improve SAM availability can be adopted. Strategies that can more finely modulate the flux of pathway metabolites, such as splitting the FA biosynthesis pathway into two modules and regulating each module separately, may unlock greater productivity of the host cells. Based on all these efforts, the widely used heterologous expression host BL21(DE3) with glucose as carbon source may still show good productivity in the heterologous biosynthesis of FA. A tyrosine over-producing strain or chromosomal integration of the biosynthesis pathway can also tested to reduce the fermentation cost. This study lacks fed-batch fermentation data, but the shake-flask results were comparable with the literature, indicating good application prospects. Our work offers a basis for further studies on the heterologous biosynthesis of phenolic natural products.

\section{Abbreviations}

FA: $p$-Hydroxy-3-methoxycinnamic acid; NADPH: Nicotinamide adenine dinucleotide phosphate; SAM: S-Adenosyl-L-methionine; HPLC: High-performance liquid chromatography.

\section{Supplementary Information}

The online version contains supplementary material available at https://doi. org/10.1186/s40643-021-00423-0.

Additional file 1: Table S1. Efforts for producing FA in E. coli. Table S2. The polymerase chain reaction (PCR) primers used in this study. Table S3. Nucleotide sequences of genes used in this study. Table S4. Effect of adding L-Methionine in FA biosynthetic pathway. Figure S1. Sodium dodecyl sulfate polyacrylamide gel electrophoresis results of TAL, SAM5 and COMT expression.

\section{Acknowledgements}

Not applicable. 


\begin{abstract}
Authors' contributions
HJL and YW conceived the project and revised the manuscript. HJL and YZ performed most of the experiment work, including the construction of the plasmids, fermentation and production detection. JS performed part of the plasmids construction at the beginning of this project. HLL established the detection method of trans-ferulic acid, $p$-coumaric acid and caffeic acid. HJL wrote the manuscript. All authors read and approved the manuscript.
\end{abstract}

\section{Funding}

This work was financially supported by the National Key Research and Development Program of China (2018YFA0900600), the Strategic Priority Research Program 'Molecular mechanism of Plant Growth and Development' of CAS (XDB27020202, XDB27020103), the National Natural Science Foundation of China (Grant Nos. 31670099, 31700261, 41876084), the Construction of the Registry and Database of Bioparts for Synthetic Biology of the Chinese Academy of Science (No. ZSYS-016), the International Partnership Program of Chinese Academy of Science (No. 153D31 KYSB20170121), Program of Shanghai Academic/Technology Research Leader (20XD1404400), the Tianjin Synthetic Biotechnology Innovation Capacity Improvement Project (TSBICIPKJGG-002-15) and the National Key Laboratory of Plant Molecular Genetics, SIPPE, CAS.

\section{Availability of data and materials}

All datasets used and analyzed in the current study are available from the corresponding author on reasonable request.

\section{Declarations}

\section{Ethics approval and consent to participate}

Not applicable.

\section{Consent for publication}

All authors agree to publish this manuscript.

\section{Competing interests}

The authors declare that they have no competing interests.

\section{Author details}

1CAS Key Laboratory of Synthetic Biology, CAS Center for Excellence in Molecular Plant Sciences, Shanghai Institute of Plant Physiology and Ecology, Chinese Academy of Sciences, Shanghai 200032, China. ${ }^{2}$ University of Chinese Academy of Sciences, Beijing 100049, China.

Received: 21 March 2021 Accepted: 28 July 2021

Published online: 10 August 2021

\section{References}

Ajikumar PK, Xiao WH, Tyo KEJ, Wang Y, Simeon F, Leonard E, Mucha O, Phon TH, Pfeifer B, Stephanopoulos G (2010) Isoprenoid pathway optimization for taxol precursor overproduction in Escherichia coli. Science 330(6000):70-74

Berner M, Krug D, Bihlmaier C, Vente A, Muller R, Bechthold A (2006) Genes and enzymes involved in caffeic acid biosynthesis in the actinomycete Saccharothrix espanaensis. J Bacteriol 188(7):2666-2673

Braga A, Faria N (2020) Bioprocess optimization for the production of aromatic compounds with metabolically engineered hosts: recent developments and future challenges. Front Bioeng Biotechnol 8:96

Cabulong RB, Valdehuesa KNG, Banares AB, Ramos KRM, Nisola GM, Lee WK, Chung WJ (2019) Improved cell growth and biosynthesis of glycolic acid by overexpression of membrane-bound pyridine nucleotide transhydrogenase. J Ind Microbiol Biotechnol 46(2):159-169

Choi O, Wu C-Z, Kang SY, Ahn JS, Uhm T-B, Hong Y-S (2011) Biosynthesis of plant-specific phenylpropanoids by construction of an artificial biosynthetic pathway in Escherichia coli. J Ind Microbiol Biotechnol 38(10):1657-1665

Cui Y-Y, Ling C, Zhang Y-Y, Huang J, Liu J-Z (2014) Production of shikimic acid from Escherichia coli through chemically inducible chromosomal evolution and cofactor metabolic engineering. Microb Cell Fact $13: 21-21$

Fowler ZL, Koffas MA (2009) Biosynthesis and biotechnological production of flavanones: current state and perspectives. Appl Microbiol Biotechnol 83(5):799-808

Goris T, Perez-Valero A, Martinez I, Yi D, Fernandez-Calleja L, San Leon D, Bornscheuer UT, Magadan-Corpas P, Lombo F, Nogales J (2020) Repositioning microbial biotechnology against COVID-19: the case of microbial production of flavonoids. Microb Biotechnol. https://doi.org/ 10.1111/1751-7915.13675

Han G, Hu X, Wang X (2016) Overexpression of methionine adenosyltransferase in Corynebacterium glutamicum for production of S-adenosylL-methionine. Biotechnol Appl Biochem 63(5):679-689

Harris PJ, Trethewey JAK (2009) The distribution of ester-linked ferulic acid in the cell walls of angiosperms. Phytochem Rev 9(1):19-33

Haslinger K, Prather KLJ (2020) Heterologous caffeic acid biosynthesis in Escherichia coli is affected by choice of tyrosine ammonia lyase and redox partners for bacterial Cytochrome P450. Microb Cell Fact 19(1):26

Heo KT, Kang SY, Hong YS (2017) De novo biosynthesis of pterostilbene in an Escherichia coli strain using a new resveratrol $O$-methyltransferase from Arabidopsis. Microb Cell Fact 16(1):30

Huang Q, Lin Y, Yan Y (2013) Caffeic acid production enhancement by engineering a phenylalanine over-producing Escherichia coli strain. Biotechnol Bioeng 110(12):3188-3196

Huang D, Yang K, Liu J, Xu Y, Wang Y, Wang R, Liu B, Feng L (2017) Metabolic engineering of Escherichia coli for the production of $2^{\prime}$-fucosyllactose and 3-fucosyllactose through modular pathway enhancement. Metab Eng 41:23-38

Jendresen CB, Stahlhut SG, Li M, Gaspar P, Siedler S, Forster J, Maury J, Borodina I, Nielsen AT (2015) Highly active and specific tyrosine ammonialyases from diverse origins enable enhanced production of aromatic compounds in bacteria and Saccharomyces cerevisiae. Appl Environ Microbiol 81(13):4458-4476

Jones KL, Kim SW, Keasling JD (2000) Low-copy plasmids can perform as well as or better than high-copy plasmids for metabolic engineering of bacteria. Metab Eng 2(4):328-338

Kabus A, Georgi T, Wendisch VF, Bott M (2007) Expression of the Escherichia coli pntAB genes encoding a membrane-bound transhydrogenase in Corynebacterium glutamicum improves L-lysine formation. Appl Microbiol Biotechnol 75(1):47-53

Kang S-Y, Choi O, Lee JK, Hwang BY, Uhm T-B, Hong Y-S (2012) Artificial biosynthesis of phenylpropanoic acids in a tyrosine overproducing Escherichia coli strain. Microb Cell Fact 11(1):153

Kunjapur AM, Hyun JC, Prather KL (2016) Deregulation of S-adenosylmethionine biosynthesis and regeneration improves methylation in the E. coli de novo vanillin biosynthesis pathway. Microb Cell Fact 15:61

Lerner CG, Inouye M (1990) Low copy number plasmids for regulated lowlevel expression of cloned genes in Escherichia coli with blue/white insert screening capability. Nucleic Acids Res 18(15):4631

Liang Y, Xi X, Liu Q, Huang P, Li J, Lin Q (2018) Research progress on the physiological activity and application of ferulic acid and its derivatives. J Food Sci Biotechnol 37(05):449-454

Lin Y, Yan Y (2012) Biosynthesis of caffeic acid in Escherichia coli using its endogenous hydroxylase complex. Microbial Cell Fact. https://doi.org/ 10.1186/1475-2859-11-42

Liu J, Li H, Zhao G, Caiyin Q, Qiao J (2018) Redox cofactor engineering in industrial microorganisms: strategies, recent applications and future directions. J Ind Microbiol Biotechnol 45(5):313-327

Luziatelli F, Brunetti L, Ficca AG, Ruzzi M (2019) Maximizing the efficiency of vanillin production by biocatalyst enhancement and process optimization. Front Bioeng Biotechnol 7:279

Ma QH, Xu Y (2008) Characterization of a caffeic acid 3-O-methyltransferase from wheat and its function in lignin biosynthesis. Biochimie 90(3):515-524

Martinez I, Zhu J, Lin H, Bennett GN, San KY (2008) Replacing Escherichia coli NAD-dependent glyceraldehyde 3-phosphate dehydrogenase (GAPDH) with a NADP-dependent enzyme from Clostridium acetobutylicum facilitates NADPH dependent pathways. Metab Eng 10(6):352-359 
Ng CY, Farasat I, Maranas CD, Salis HM (2015) Rational design of a synthetic Entner-Doudoroff pathway for improved and controllable NADPH regeneration. Metab Eng 29:86-96

Ni J, Tao F, Du H, Xu P (2015) Mimicking a natural pathway for de novo biosynthesis: natural vanillin production from accessible carbon sources. Sci Rep $5: 13670$

Noronha SB, Yeh HJ, Spande TF, Shiloach J (2000) Investigation of the TCA cycle and the glyoxylate shunt in Escherichia coli BL21 and JM109 using (13) C-NMR/MS. Biotechnol Bioeng 68(3):316-327

Rodrigues JL, Araujo RG, Prather KL, Kluskens LD, Rodrigues LR (2015a) Heterologous production of caffeic acid from tyrosine in Escherichia coli. Enzyme Microb Technol 71:36-44

Rodrigues JL, Araujo RG, Prather KL, Kluskens LD, Rodrigues LR (2015b) Production of curcuminoids from tyrosine by a metabolically engineered Escherichia coli using caffeic acid as an intermediate. Biotechnol J 10(4):599-609

Rodrigues JL, Gomes D, Rodrigues LR (2020) A combinatorial approach to optimize the production of curcuminoids from tyrosine in Escherichia coli. Front Bioeng Biotechnol 8:59

Sauer U, Canonaco F, Heri S, Perrenoud A, Fischer E (2004) The soluble and membrane-bound transhydrogenases UdhA and PntAB have divergent functions in NADPH metabolism of Escherichia coli. J Biol Chem 279(8):6613-6619

Serra S, Fuganti C, Brenna E (2005) Biocatalytic preparation of natural flavours and fragrances. Trends Biotechnol 23(4):193-198

Sgarbossa A, Giacomazza D, di Carlo M (2015) Ferulic acid: a hope for Alzheimer's disease therapy from plants. Nutrients 7(7):5764-5782

Shiloach J, Kaufman J, Guillard AS, Fass R (1996) Effect of glucose supply strategy on acetate accumulation, growth, and recombinant protein production by Escherichia coli BL21 (DE3) and Escherichia coli JM109. Biotechnol Bioeng 49(4):421-428

Wang Y, Boghigian BA, Pfeifer BA (2007) Improving heterologous polyketide production in Escherichia coli by overexpression of an S-adenosylmethionine synthetase gene. Appl Microbiol Biotechnol 77(2):367-373

Wang J-F, Xiong Z-Q, Li S-Y, Wang Y (2013) Enhancing isoprenoid production through systematically assembling and modulating efflux pumps in Escherichia coli. Appl Microbiol Biotechnol 97(18):8057-8067
Wang J-F, Zhang S-L, Wang Y (2014) Pathway assembly and optimization in E. coli for de novo biosynthesis of resveratrol. China Biotechnol 34(2):71-77

Watts KT, Lee PC, Schmidt-Dannert C (2004) Exploring recombinant flavonoid biosynthesis in metabolically engineered Escherichia coli. ChemBioChem 5(4):500-507

Wen Y, Ushio H (2017) Ferulic acid promotes hypertrophic growth of fast skeletal muscle in zebrafish model. Nutrients. https://doi.org/10.3390/ nu9101066

Yamauchi Y, Hirasawa T, Nishii M, Furusawa C, Shimizu H (2014) Enhanced acetic acid and succinic acid production under microaerobic conditions by Corynebacterium glutamicum harboring Escherichia coli transhydrogenase gene pntAB. J Gen Appl Microbiol 60(3):112-118

Zhang C, Hong K (2020) Production of Terpenoids by Synthetic Biology Approaches. Front Bioeng Biotechnol. https://doi.org/10.3389/fbioe.2020. 00347

Zhao C, Zhao Q, Li Y, Zhang Y (2017) Engineering redox homeostasis to develop efficient alcohol-producing microbial cell factories. Microb Cell Fact 16(1):115

Zhou JM, Gold ND, Martin VJ, Wollenweber E, Ibrahim RK (2006) Sequential $O$-methylation of tricetin by a single gene product in wheat. Biochim Biophys Acta 1760(7):1115-1124

Zhou J-M, Seo YW, Ibrahim RK (2009) Biochemical characterization of a putative wheat caffeic acid O-methyltransferase. Plant Physiol Biochem 47(4):322-326

Zocchi A, Jobe AM, Neuhaus JM, Ward TR (2003) Expression and purification of a recombinant avidin with a lowered isoelectric point in Pichia pastoris. Protein Expr Purif 32(2):167-174

\section{Publisher's Note}

Springer Nature remains neutral with regard to jurisdictional claims in published maps and institutional affiliations.

\section{Submit your manuscript to a SpringerOpen ${ }^{\odot}$ journal and benefit from:}

- Convenient online submission

- Rigorous peer review

- Open access: articles freely available online

- High visibility within the field

- Retaining the copyright to your article

Submit your next manuscript at $\boldsymbol{\nabla}$ springeropen.com 